\title{
Correction to: Validation of central serous chorioretinopathy multimodal imaging-based classification system
}

\author{
Jay Chhablani $^{1}$ [D . Francine Behar-Cohen ${ }^{2}$ on behalf of Central Serous Chorioretinopathy International Group
}

Published online: 6 November 2021

(c) Springer-Verlag GmbH Germany, part of Springer Nature 2021

\section{Correction to: Graefe's Archive for Clinical and Experimental Ophthalmology https://doi.org/10.1007/s00417-021-05452-1}

In the original published article, the individual collaborator names were not captured under the group name "CSCR International Group".

The names are listed below:

Pauline Aymard

Talal Beydoun

Elodie Bousquet

Francine Behar-Cohen

Chadi Mehanna

Jay Chhablani

Chui Ming Gemmy Cheung

Alejandra Daruich

K. Bailey Freund

Alain Gaudric

Camiel J.F. Boon

Andrew Lotery

Marco Lupidi

Irmela Mantel

Thibaud Mathis

Alexandre Matet

Martine Mauget-Faÿsse

Sarah Mrejen

Giuseppe Querques

Jorge Ruiz-Medrano
Jose-Maria Ruiz-Moreno

Shiri Shulman

Sumit Randhir Singh

Sobha Sivaprasad

Richard F. Spaide

Elon.H.C van Dijk

Suzanne Yzer

Min Zhao

Sandrine Zweifel

This is being corrected in this publication.

Publisher's note Springer Nature remains neutral with regard to jurisdictional claims in published maps and institutional affiliations.

The original article can be found online at https://doi.org/10.1007/ s00417-021-05452-1.

Jay Chhablani

jay.chhablani@gmail.com

1 Department of Ophthalmology, UPMC Eye Center,

University of Pittsburgh Eye and Ear Institute, Pittsburgh,

PA, USA

2 Ophtalmopole, Cochin Hospital, University of Paris, Paris, France 